\title{
Reconfigurable diplexer based on switched delay line approach
}

\author{
Zhurbenko, Vitaliy; Acar, Öncel; Ruaro, Andrea; Johansen, Tom Keinicke
}

Published in:

2013 SBMO/IEEE MTT-S International Microwave and Optoelectronics Conference (IMOC)

Link to article, DOI:

10.1109/IMOC.2013.6646447

Publication date:

2013

Link back to DTU Orbit

Citation (APA):

Zhurbenko, V., Acar, Ö., Ruaro, A., \& Johansen, T. K. (2013). Reconfigurable diplexer based on switched delay line approach. In 2013 SBMO/IEEE MTT-S International Microwave and Optoelectronics Conference (IMOC) IEEE. https://doi.org/10.1109/IMOC.2013.6646447

\section{General rights}

Copyright and moral rights for the publications made accessible in the public portal are retained by the authors and/or other copyright owners and it is a condition of accessing publications that users recognise and abide by the legal requirements associated with these rights.

- Users may download and print one copy of any publication from the public portal for the purpose of private study or research

- You may not further distribute the material or use it for any profit-making activity or commercial gain

- You may freely distribute the URL identifying the publication in the public portal

If you believe that this document breaches copyright please contact us providing details, and we will remove access to the work immediately and investigate your claim 


\title{
Reconfigurable Diplexer Based on Switched Delay Line Approach
}

\author{
Vitaliy Zhurbenko, Öncel Acar, Andrea Ruaro, Tom K. Johansen \\ Technical University of Denmark \\ Kgs. Lyngby, Denmark \\ vz/oncac/ruaro/tkj@elektro.dtu.dk
}

\begin{abstract}
In this work, a concept for the realization of a reconfigurable microwave multiplexer based on a switched delayline topology is presented. The multiplexing concept is studied by considering a diplexer example. The topology provides two times reduction in the number of filters in comparison to the conventional directional filter approach. The switched delay-line topology enables the lossy and nonlinear switching elements to be used as a part of the coupling elements rather than within the resonators. Therefore, the diplexer potentially allows for a low insertion loss and high linearity. In addition, independent tuning of channels can be achieved.
\end{abstract} line.

Keywords-microwave diplexer; multiplexer; switched delay-

\section{INTRODUCTION}

The use of reconfigurable transceivers potentially offers a great reduction in price of terrestrial and satellite systems for communication, broadcasting, and other services. This economical concept, however, requires on-the-fly frequency relocation. Dynamic range requirements for communication and broadcasting systems make radio frequency frontend multiplexers indispensable. These multiplexers and filters are required to protect receivers from out-of-band interfering signals (i.e., from transmit signals of the same or other services) and protect transmitters from polluting the electromagnetic spectrum. Therefore, reconfigurable multiplexers are key building blocks for flexible communication systems.

Since other frontend components such as switches, amplifiers, and mixers can be designed for ultra-wideband operation [1]-[5], reconfiguration of transceivers is generally limited by the reconfiguration capabilities of multiplexers. Therefore, reconfigurable multiplexers will be the key enablers of future flexible communication systems.

The advantages offered by reconfigurable multiplexers (such as mass and volume reduction as well as total cost reduction of the system) attracted a great deal of interest from microwave community. Even though, most of the research in the field of reconfigurable front-end components is focused on RF filters rather than multiplexers [6]-[9], yet reconfigurable filters is still considered as an immature technology [10]. This is mainly due to the limitations of tuning components such as varactors, BST capacitors, and others (high non-linearity, low $\mathrm{Q}$, etc.) [11]. The reconfiguration concept proposed in this paper is based on switched delay-line resonators, which, according to the original paper [13], does not rely on highquality components.

The block diagram of the considered diplexer is shown in Fig. 1.

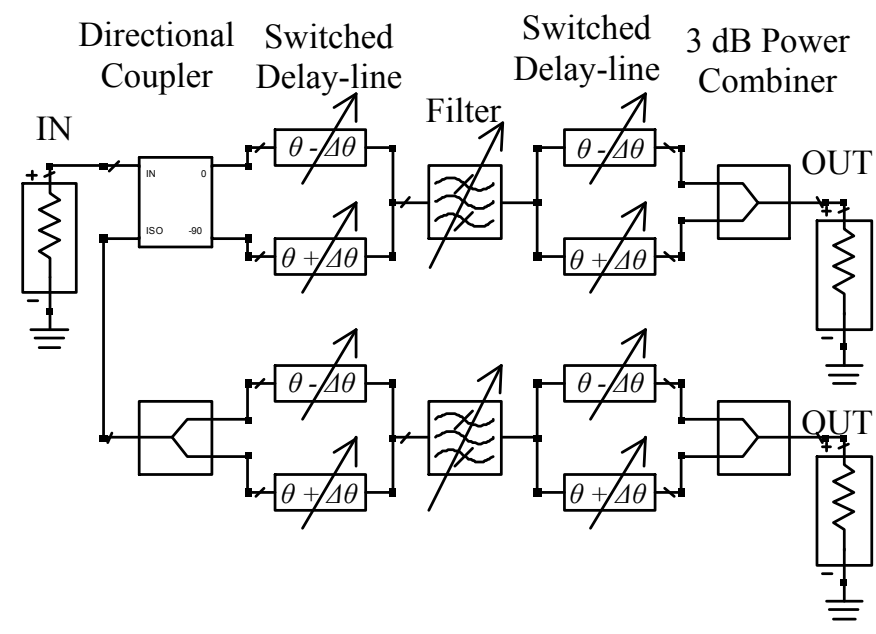

Fig. 1. Diagram of the diplexer.

Each branch of the diplexer contains two switched delayline resonators consisting of a $3 \mathrm{~dB}$ power divider (or a directional coupler) and a delay line. These resonators are connected through unit elements which are usually filters, which improve both the shape factor and stop-band rejection of the diplexer. An example employing a simple coupled line resonator and two transmission line impedance transformers as a unit element is shown in Fig. 2.

This architecture allows independent tuning of channels and requires only one filter as compared to the traditional directional filter approach [12], where two identical filters are usually used for each channel.

\section{OPERATION PRINCIPle of The SWitched Delay-Line RESONATOR}

The background theory of the switched delay-line resonator can be found in the literature [13]-[15]. Here we present only a 


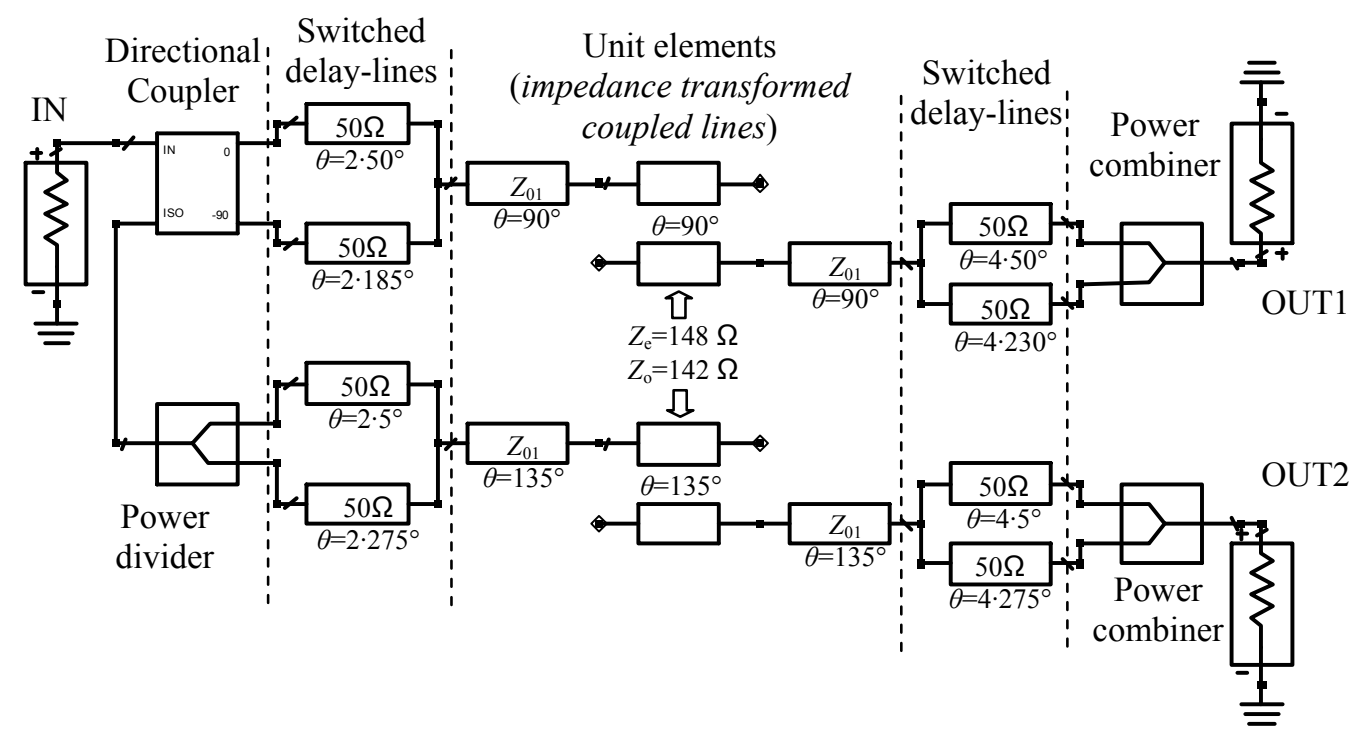

Fig. 2. Example of a diplexer based on a switched delay-line approach. $\mathrm{Z}_{01}=\operatorname{sqrt}(0.12) \cdot 50 / 2 \Omega . \theta$ are given at $f_{0}=11 \mathrm{GHz}$.

short description of the resonator, required to understand the overall tunable diplexer concept. As one can see from the diagram of the switched delay-line resonator in Fig. 3, the incoming signal enters a $3-\mathrm{dB}$ power divider and propagates along two parallel delay lines. A multi-pole RF switch combines the signal again at the output.

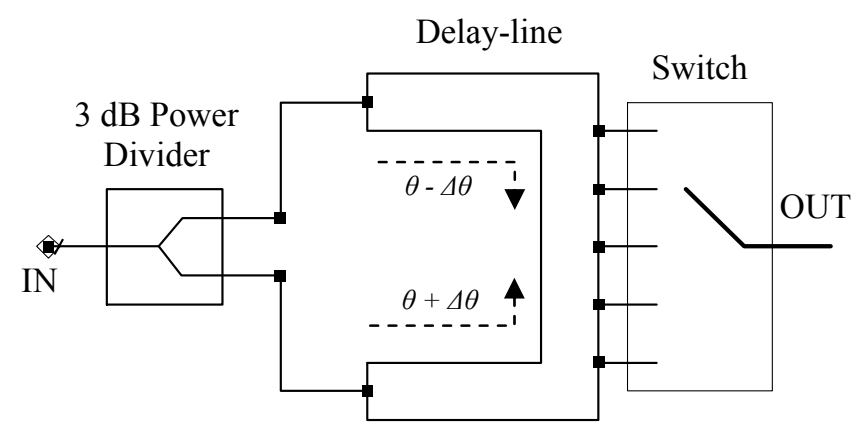

Fig. 3. Switched delay-line resonator [13]. The signals from two parallel delay lines are combined at the output with adjustable phase difference $2 \Delta \theta$. This phase difference defines the frequency response of the circuit.

The transmission response is entirely determined by the phase relationship between the delay lines, where band-stop and band-pass frequencies occur at $n f_{0} / 2$, for odd $n$, and $m f_{0} / 2$, for even $m$, respectively. Here $f_{0}$ is the center of the first passband. The relative phase between the two delay lines, $2 \Delta \theta$, can be expressed in the following way: $\Delta \theta=\pi f / f_{0}$.

A generic unit element which follows the switched delay line resonator in Fig. 2 can be either a simple transmission line, an impedance-transformed coupled line section (like shown in Fig. 2), or a higher order tunable filter, as it will be demonstrated later in Section IV.

\section{DIPLEXER ARCHITECTURE DISCUSSIONS}

Generally, there are three traditional fixed frequency multiplexing methods: (1) circulator/filter chain, (2) directional filter approach, and (3) manifold multiplexing technique [12].

The main advantage of the first multiplexing method is that the existing system can be easily extended for additional services (channels). The significant drawback of this method is the power loss of the individual channels caused by the present circulator. Furthermore, power-handling capabilities and dissipated power must be carefully considered. In relation to the current application, where frequency tuning is involved, the bandwidth of the implemented circulators must be considered. If the circulator exhibits narrowband behavior, the overall tuning capabilities of the multiplexer will be limited by the bandwidth of the circulator.

Multiplexing using the second (directional filter) approach [16] provides the same features as the method with circulator/filter modules. Further advantages, as opposed to the circulator/filter approach, are lower insertion loss and higher power-handling capability. The essential drawback of the directional filter multiplexing method results from the required hardware effort (the conventional design requires two filters and two hybrids for each channel, as shown in Fig. 4) resulting in high mass and large size.

The main advantage of the third, manifold multiplexing, method, in contrast to the above mentioned modular concepts, are low insertion loss, compact size, low mass and high powerhandling capability. Due to the mentioned advantages, this method is one of the most popular multiplexing methods for space applications. With regard to the considered here tunable application, the main drawback is the high interaction of the channel filters where the situation of degraded in-band performance is likely to occur.

The multiplexing approach presented in this work resembles the directional filter architecture presented in Fig. 4 in the sense that the directional coupler is used to split the frequency channels. The proposed topology, however, requires only one filter per channel (typically, such multiplexers would require two identical filters, as it was outlined above) and a 
power combiner can be used at the output instead of directional coupler if preferred. (refer to Fig. 1 and Fig. 4). The filter reconfiguration approach based on the described above switched delay-line is extended forming a multiplexer.

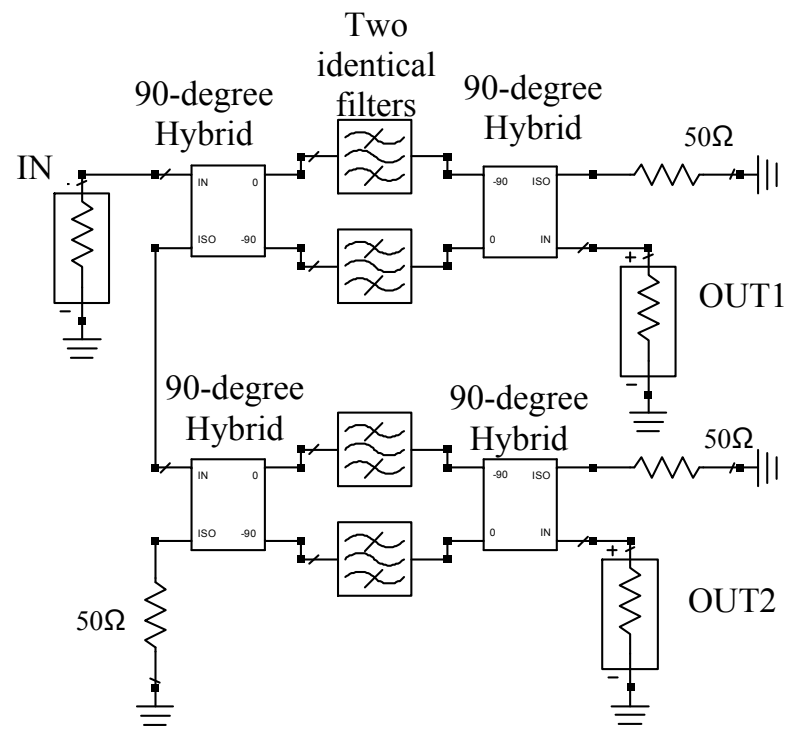

Fig. 4. Fixed frequency diplexer based on directional filter approach. The reflected wave in the first channel is directed to the second channel due to 90 degrees phase shift in hybrides. The approach requires two identical filters in each diplexer channel.

The input power divider is substituted by a directional coupler further extending the filter, as it is shown in Fig. 1 and Fig. 2 in the case of a diplexer (for more channels the subsequent power divider is replaced with a directional coupler).

The broadband response of the diplexer is shown in Fig. 5.

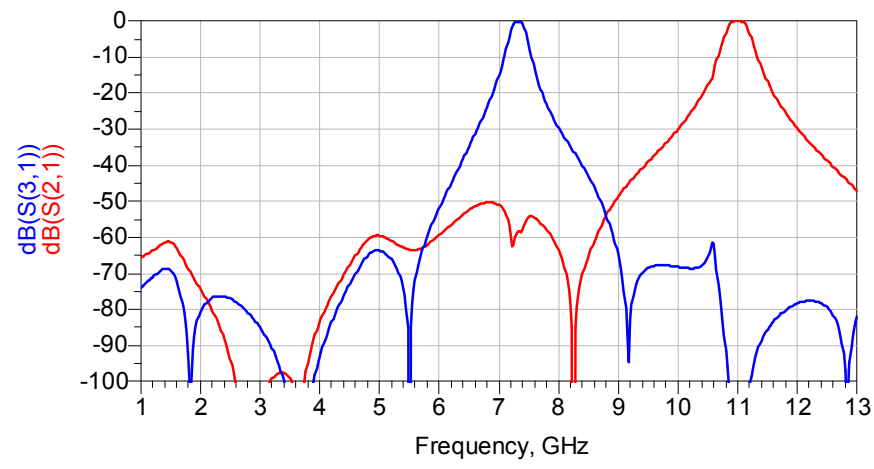

Fig. 5. Broadband response of the diplexer in Fig. 2.

The filtering properties of the diplexer are defined by a filter in each branch. A simple coupled line is used as a filter in this example, but a higher order filter can be implemented according to overall multiplexer specification, as it will be demonstrated later.
Reconfiguration is achieved by changing the length of the transmission lines (delay lines) [13] and tuning the channel filter itself. In this manner each channel can be tuned individually, as it is shown in Fig. 6.

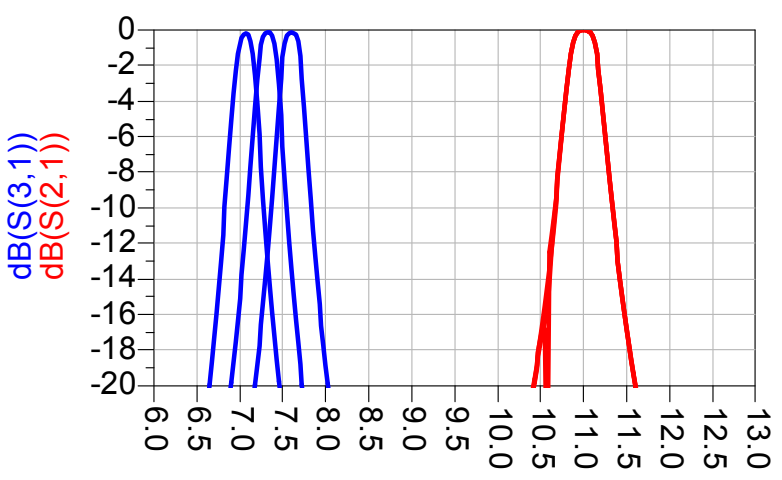

Frequency, $\mathrm{GHz}$

(a)

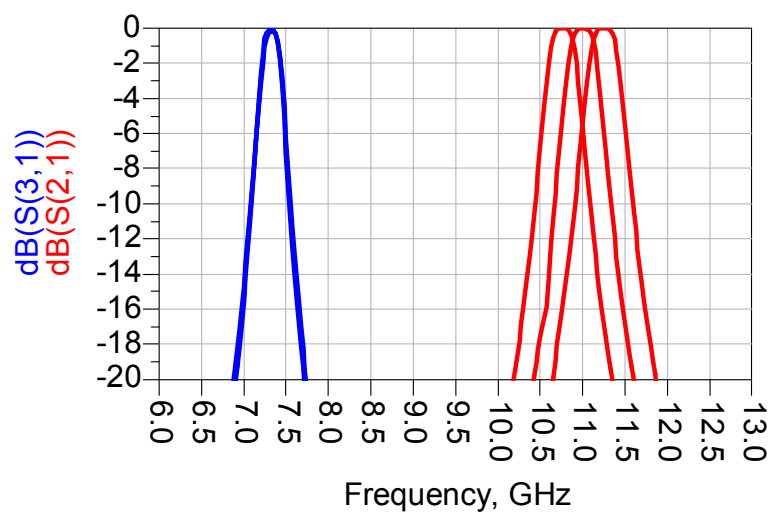

(b)

Fig. 6. Tuning diplexer in Fig. 2. Indepandent tuning of channels can be achieved.

As one can see from the presented data, the considered architecture allows for independent tuning of the channels.

There are applications where a closer distance between the channels is required. The example featuring this is considered in the following Section.

\section{IMPROVING THE SHAPE FACTOR OF SWITCHED DELAY LINE DIPLEXER}

There are many high demanding applications where a more selective behavior is required. In order to improve the shape factor of the diplexer, a higher order filter can be introduced in the unit element. An example of such a diplexer is shown in Fig. 7. Each filter consists of five identical coupled line sections. As it was mentioned before, the power dividers in Fig. 1 can be replaced by couplers. This has been implemented in the diplexer shown in Fig. 7. This substitution also reduces the variety of implemented components, which can be an advantage in practical implementation of the diplexer. Instead of designing a directional coupler and a 


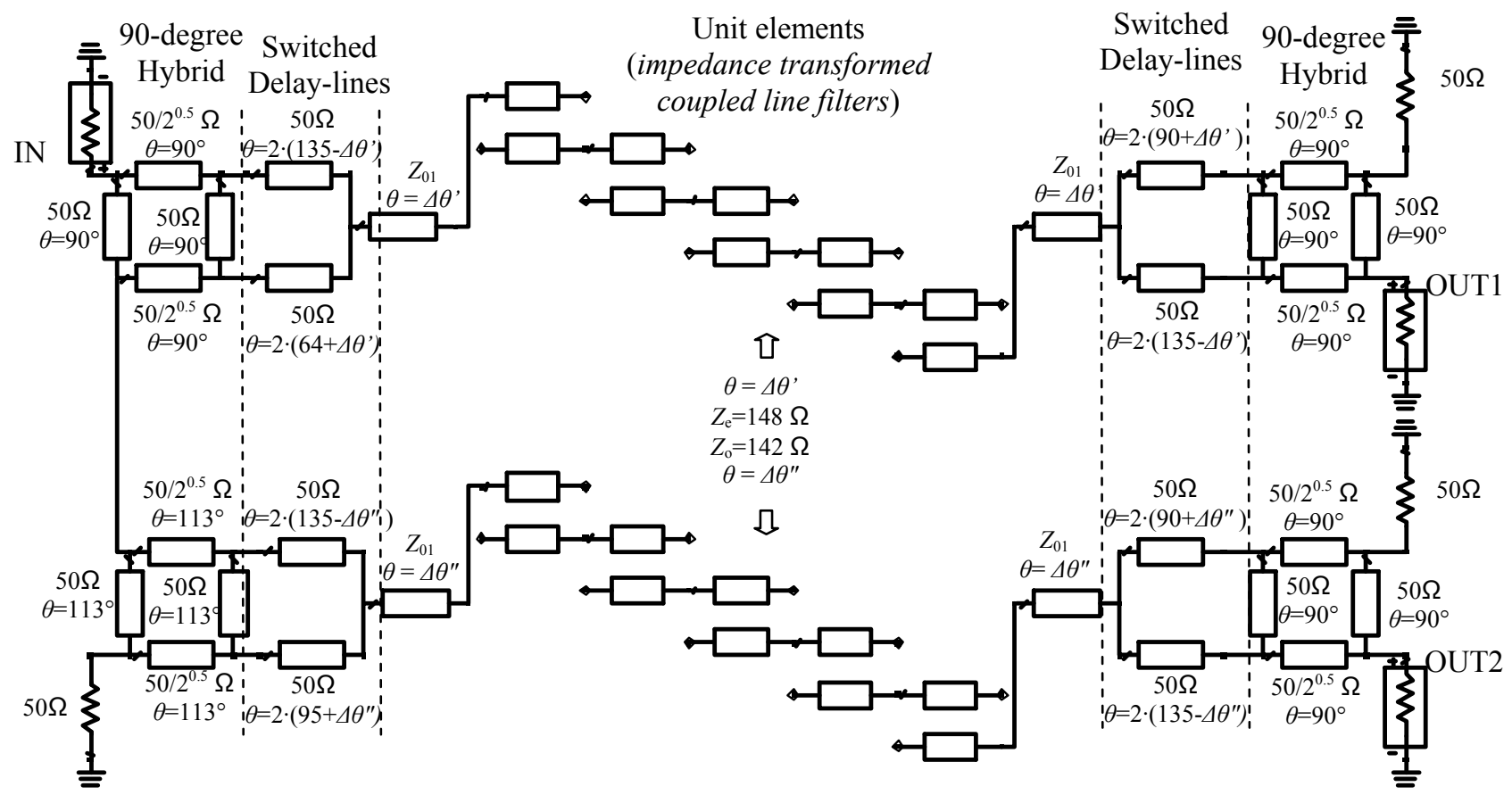

Fig. 7. Example of a diplexer using higher order filters. The switching of the delay lines and filters corresponds to various values of $\Delta \theta^{\prime}$ and $\Delta \theta^{\prime \prime} . \mathrm{Z}_{01}=\operatorname{sqrt}(0.12) \cdot 50 / 2 \Omega . \theta$ are given at $f_{0}=11 \mathrm{GHz}$.

power divider, one would just need to design only a directional coupler, which might reduce the design efforts. A conventional 90-degree coupler is implemented in this design, which consists of four quarter-wave transmission line sections. Since such a coupler is relatively narrowband, the dimensions of the coupler at the second channel have to be adjusted for optimal operation at the corresponding center frequency of the second channel. The electrical lengths of the components in Fig. 7 are given at the center frequency of the first channel of the diplexer, $11 \mathrm{GHz}$. The original implementation of the switched delay-line resonator is based on a conventional power divider which splits the input signal in two with equal amplitudes and phases. Since the implemented coupler inherently introduces 90 degrees phase shift between the output terminals, the electrical length of a delay line connected to the corresponding terminal is shortened. The values for the parameters of all the components are given in Fig. 7. The switching of the delay lines and filters is simulated using various values of $\Delta \theta^{\prime}$ and $\Delta \theta^{\prime \prime}$. One of the examples describing the way the switching is done in practice can be found in the references [6], [13]-[15].

Already with this simple and not optimized design one can see from data in Fig. 8 how the higher order filter shapes the response, preserving the tuning (also refer to Fig. 9...Fig. 10). Thus, it is possible to use an arbitrary unit element that can provide the required shape factor. The drawback in this case is the need to design and implement a higher order filter for every switching position, that takes considerably more space, and that is likely to have higher losses.

Here, three reconfiguration scenarios are considered: both channels are tuned simultaneously (Fig. 8), channel one is fixed while channel two is tuned (Fig. 9), and channel one is tuned while channel two is fixed (Fig. 10).

In the first scenario $\Delta \theta^{\prime}$ (refer to Fig. 6) changes from $87.9^{\circ}$ to $91.8^{\circ}$, and $\Delta \theta^{\prime \prime}$ changes from $92.9^{\circ}$ to $96.8^{\circ}$. The achieved response of the diplexer is shown in Fig. 8.

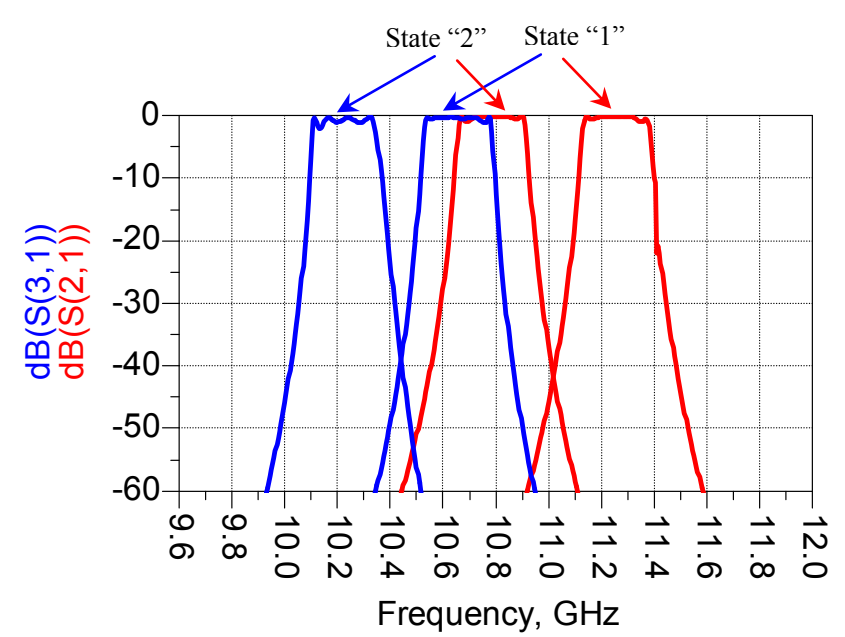

Fig. 8. Using a 5th order coupled line band pass filter as the unit element. Improved shape factor and stopband rejection are achieved.

In the second scenario $\Delta \theta^{\prime}$ is fixed at $90^{\circ}$ (refer to Fig. 6), while $\Delta \theta^{\prime \prime}$ changes from $94^{\circ}$ to $96.5^{\circ}$. The achieved response of the diplexer is shown in Fig. 9. 


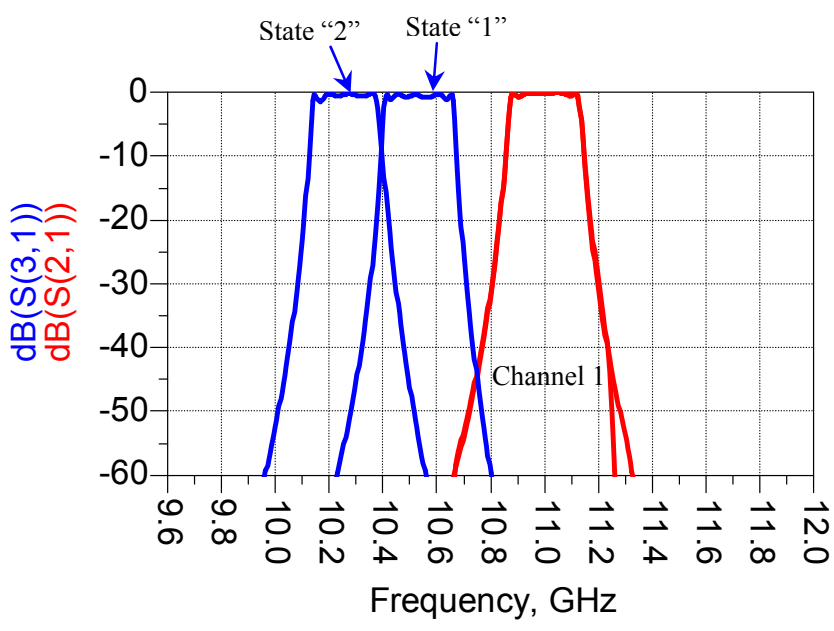

Fig. 9. Tuning the 5th order coupled line band pass filter. Channel one is fixed.

Finally, in the third scenario, $\Delta \theta^{\prime \prime}$ is fixed at $95^{\circ}$ while $\Delta \theta^{\prime}$ changes from $88.6^{\circ}$ to $90.9^{\circ}$. The achieved response of the diplexer is shown in Fig. 10.

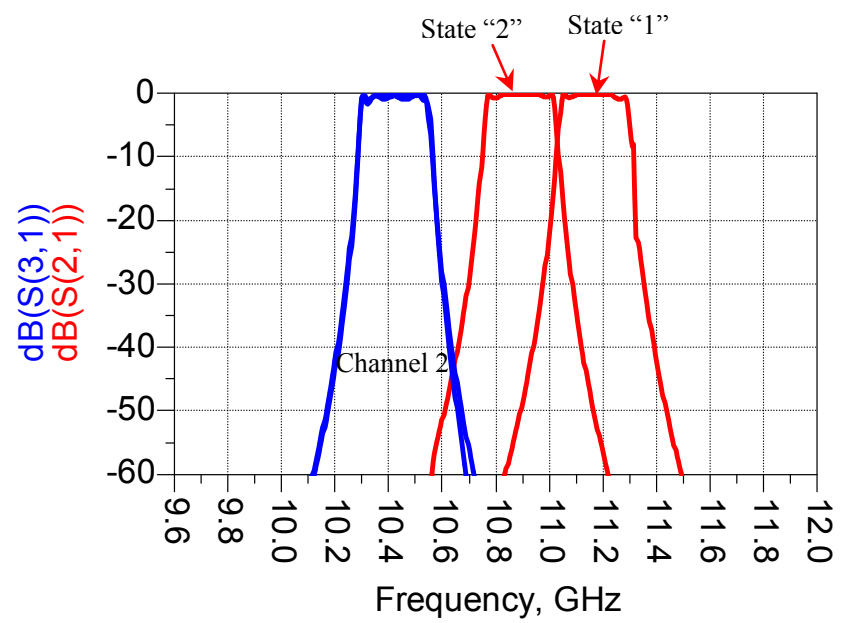

Fig. 10. Tuning the 5th order coupled line band pass filter. Channel two is fixed.

As one can see from the presented data, simultaneous (Fig. 8) and independent ((Fig. 9 and Fig. 10) reconfiguration of the channels can be achieved.

It should be mentioned that the implemented narrowband directional couplers limit the tuning range of the diplexer. In fact, the demonstrated in Fig. 8...Fig. 10 data represents the maximum achievable tuning range for the circuit in Fig. 7. Further tuning leads to degraded performance of the diplexer. More broadband realizations of the directional couplers should be considered if a wider tuning range is required.

\section{CONCLUSION}

In this work, it was shown that the filter based on the switched delay-line approach can be extended to form a multiplexer topology. The design of a diplexer is shown here as an example. The considered topology requires only one filter per channel as opposed to typical directional topologies where two identical filters are typically required. In addition, a power combiner can be used at the output instead of directional coupler if preferred.

It is shown that the selectivity of the switched delay-line diplexer can be further improved implementing higher order filters as the unit elements.

\section{REFERENCES}

[1] V. Zhurbenko, "A High-isolation Switch Based on a Standard GaAs Process," Microwave and Optical Technology Letters, vol. 53, no. 5, pp. $984-987,2011$.

[2] T. Johansen, V. Krozer, J. Vidkjær, "Design and Analysis of Wide-band SiGe HBT Active Mixers," IEEE Trans. Microwave Theory and Techniques, vol. 53, issue 7, pp. 2389-2397, 2005.

[3] K. Narendra, E. Limiti, C. Paoloni, V. Zhurbenko, "pHEMT Distributed Power Amplifier Employing Broadband Impedance Transformer," Microwave Journal, Vol. 56, No. 6, June 2013, pp. 76-82.

[4] L. Yan, V. Krozer, S. Delcourt, V. Zhurbenko, T. K. Johansen, and C. Jiang, "GaAs Wideband Low Noise Amplifier Design for Breast Cancer Detection System," Proceedings of the 2009 Asia Pacific Microwave Conference, Singapore, pp. 1-4, 2009.

[5] Advanced Microwave Circuits and Systems, edited by V. Zhurbenko, In-Tech, 2010, p. 490.

[6] P. W. Wong, and I. Hunter, "Electronically Tunable Filters," IEEE Microwave Magazine, October 2009, pp. 46-54.

[7] E. J. Naglich, J. Lee, D. Peroulis, and W. J. Chappell, " Extended Passband Bandstop Filter Cascade With Continuous $0.85-6.6-\mathrm{GHz}$ Coverage," IEEE Transactions on Microwave Theory and Techniques, Vol. 60, No. 1, January 2012, pp. 21-30.

[8] J. S. Sun, N. Kaneda, Y. Baeyens, T. Itoh, and Young-Kai Chen, " Multilayer Planar Tunable Filter With Very Wide Tuning Bandwidth," IEEE Transactions on Microwave Theory and Techniques, Vol. 59, No. 11, November 2011, pp. 2864-2871.

[9] Passive Microwave Components and Antennas, edited by V. Zhurbenko, In-Tech, 2010, p. 556.

[10] Y. Neuvo, "Unfogging the Future," presented at the European Microwave Week 2012, Amsterdam, The Netherlands, 29 October 1 November 2012.

[11] C. Huang, K. Buisman, L. Nanver, and L. de Vreede, \The state-of-theart of RF capacitive tunable components," Solid-State and Integrated Circuit Technology (ICSICT), 2010 10th IEEE International Conference on, 112010 , pp. 619-622.

[12] Waveguide Components for Antenna Feed Systems by Jaroslav Uher et al., Artech House, Boston-London, 1993, p. 457.

[13] P. W. Wong, and I. C. Hunter, "A New Class of Low-Loss HighLinearity Electronically Reconfigurable Microwave Filter," IEEE Transactions on Microwave Theory and Techniques, Vol. 56, No. 8, August 2008, pp. 1945-1953.

[14] P. W. Wong and I. C. Hunter, "Electronically Reconfigurable Microwave Bandpass Filter," IEEE Transactions on Microwave Theory and Techniques, Vol. 57, No. 12, December 2009, pp. 3070-3079.

[15] P. W.Wong and I. C. Hunter, "Parallel-coupled switched delay line (SDL) reconfigurable microwave Filter", IEEE MTT-S Int. Microwave Symp. Dig., June 2009, pp.513 -516.

[16] Mobbs et. al., "The Use of Matched Four-Port Filters to Realize Switched Multiplexers with having low amplitude and group-delay ripple” , IEEE-MTT, vol. 12, no. 12, pp. 1183-1191, 1987. 